\title{
Cesàro and Abel ergodic theorems for integrated semigroups
}

https://doi.org/10.1515/conop-2020-0119

Received February 15, 2021; accepted September 7, 2021

Abstract: Let $\{S(t)\}_{t \geq 0}$ be an integrated semigroup of bounded linear operators on the Banach space $X$ into itself and let $A$ be their generator. In this paper, we consider some necessary and sufficient conditions for the Cesàro mean and the Abel average of $S(t)$ converge uniformly on $\mathcal{B}(X)$. More precisely, we show that the Abel average of $S(t)$ converges uniformly if and only if $\mathcal{X}=\mathcal{R}(A) \oplus \mathcal{N}(A)$, if and only if $\mathcal{R}\left(A^{k}\right)$ is closed for some integer $k$ and $\left\|\lambda^{2} R(\lambda, A)\right\| \longrightarrow 0$ as $\lambda \rightarrow 0^{+}$, where $\mathcal{R}(A), \mathcal{N}(A)$ and $R(\lambda, A)$, be the range, the kernel, the resolvent function of $A$, respectively. Furthermore, we prove that if $S(t) / t^{2} \longrightarrow 0$ as $t \rightarrow \infty$, then the Cesàro mean of $S(t)$ converges uniformly if and only if the Abel average of $S(t)$ is also converges uniformly.

Keywords: Cesàro means, Abel averages, Integrated semigroups, Uniform Abel ergodic, Uniform Cesàro ergodic.

MSC: 47D62

\section{Introduction}

Throughout this paper $\mathcal{B}(X)$ denotes the Banach algebra of all bounded linear operators on Banach space $X$ into itself. Let $A$ be a closed linear operator on $X$ with domain $D(A) \subset X$, we denote by $\mathcal{N}(A), \mathcal{R}(A), \sigma(A)$, $\rho(A)$ and $R(., A)$, the kernel, the range, the spectrum, the resolvent set and the resolvent operator of $A$, respectively.

The family $\{T(t)\}_{t \geq 0}$ of bounded linear operator on $X$ is called a strongly continuous semigroup $\left(C_{0^{-}}\right.$ semigroup in short [2]) if it has the following properties:

1. $T(0)=I$,

2. $T(t) T(s)=T(t+s)$,

3. The map $t \rightarrow T(t) x$ from $[0,+\infty[$ into $X$ is continuous for all $x \in X$.

Their infinitesimal generator $A$ is defined by:

$$
A x=\lim _{t \rightarrow 0^{+}} \frac{T(t) x-x}{t} \text { for all } x \in D(A),
$$

where

$$
D(A)=\left\{x \in X: \lim _{t \rightarrow 0^{+}} \frac{T(t) x-x}{t} \text { exists }\right\}
$$

The Laplace transformation $R(\lambda)$ of a $C_{0}$-semigroup $T(t)$ on $\mathcal{B}(X)$, defined as

$$
R(\lambda) x=\int_{0}^{\infty} e^{-\lambda t} T(t) x d t,
$$

\footnotetext{
^Corresponding Author: Fatih Barki: Sidi Mohamed Ben Abdellah University. Faculty of Sciences Dhar El Mahraz. Fez, Mo-
} rocco. Mathematical Sciences and Applications Laboratory, E-mail: fatih.barki@usmba.ac.ma 
which is exactly the resolvent function of $A$. Moreover, the infinitesimal generator of a $C_{0}$-semigroup is a linear closed densely defined operator on a Banach space $X$, see for instance [12] and [3].

Integrated semigroups and $n$-time Integrated semigroups, $n \in \mathbb{N}$, of operators in Banach space were introduced by Arendt [1] and studied by Arendt, Kellermann, Hieber [7], Thieme [17] and many others.

A relevant example is obtained if we assume that $\{T(t)\}_{t \geqslant 0}$ is a $C_{0}$-semigroup of bounded linear operator on $X$, then $S(t)=\int_{0}^{t} T(r) d r$ defines an integrated semigroup $\{S(t)\}_{t \geqslant 0}$ having the following three properties:

1. $S(0)=0$,

2. $S(s) S(t)=\int_{0}^{s} S(r+t)-S(r) d r$ for $t, s \geq 0$,

3. The map $t \rightarrow S(t)$ from $[0,+\infty$ [ into $X$ is strongly continuous.

Arendt [1] showed that certain natural classes of operators, such as adjoint semigroups of $C_{0}$-semigroups on non-reflexive Banach spaces, give rise to integrated semigroups which are not integrals of $C_{0}$-semigroups. In contrast to $C_{0}$-semigroups, integrated semigroups may be not exponentially bounded, may be locally defined, and their generators may be not densely defined.

Ergodic theorems have a long tradition and are usually formulated in semigroup theory via the existence of limits of the Cesàro means, defined by:

$$
\mathcal{e}(t)=t^{-1} \int_{0}^{t} T(s) d s, \text { for } t \geq 0
$$

where $\{T(t)\}_{t \geq 0} \subset \mathcal{B}(X)$ is a $C_{0}$-semigroup. In this case, $\{T(t)\}_{t \geq 0}$ is said to be uniformly Cesàro ergodic if the Cesàro means of $T(t)$ converges uniformly in $\mathcal{B}(X)$, as $t \rightarrow \infty$. This notion is completely connected to study the limit of the Abel averages of a $C_{0}$-semigroup $\{T(t)\}_{t \geq 0}$, defined as:

$$
\lambda R(\lambda, A)=\lambda \int_{0}^{\infty} e^{-\lambda t} T(t) x d t .
$$

where $A$ is the generator of $\{T(t)\}_{t \geq 0}$ and $R(\lambda, A)$ is the resolvent function of $A$.

Recall that, a $\mathrm{C}_{0}$-semigroup $T(t)$ is called uniformly Abel ergodic if the limit, as $\lambda \rightarrow 0^{+}$, if their Abel averages exists in the norm operator topology.

We will denote the growth bound of a $C_{0}$-semigroup $\{T(t)\}_{t \geq 0}$ by

$$
\omega_{0}=\inf \left\{w \in \mathbb{R}: \text { there exists } M \text { such that }\|T(t)\| \leq M e^{w t}, t \geq 0\right\} .
$$

Usually one assumes $\omega_{0} \leq 0$ or the even stronger condition $\|T(t)\| / t \longrightarrow 0$ as $t \rightarrow \infty$, to study the convergence of the Cesàro means and the Abel averages of $\{T(t)\}_{t \geq 0}$. Generally, great attention has been focused on the study the relationship between Cesàro ergodicity and Abel ergodicity for different classes of semigroups in $\mathcal{B}(X)$. The result of Hille and Phillips in [6, Theorem 18.8.4] deals with the uniform Abel ergodicity of semigroups of class $(A)$, a class slightly larger than $C_{0}$-semigroups, under the assumption $\omega_{0} \leq 0$. More precisely, they have shown that $T(t)$ is uniformly Abel ergodic if and only if $\lambda^{2} R(\lambda, A) x \longrightarrow 0$ as $\lambda \rightarrow 0^{+}$for every $x \in X$ and $\mathcal{X}=\mathcal{R}(A) \oplus \mathcal{N}(A)$. Furthermore, if $T(t)$ is uniformly Abel ergodic, then $\mathcal{R}\left(A^{m}\right)=\mathcal{R}(A)$, for all $m \in \mathbb{N}^{\star}$. A relevant result obtained by S.Y. Shaw in [13] for a locally integrated semigroup, under an assumption weaker than $\omega_{0} \leq 0$, that means $T(t)$ is uniformly Cesàro ergodic if and only if it satisfies the following conditions:

(i) The Laplace transformation $R_{\lambda}$ exists for every $\lambda>0$,

(ii) $\left\|T(t) R_{\lambda}\right\| / t \longrightarrow 0$ as $t \rightarrow \infty$, for some $\lambda>0$, and

(iii) $T(t)$ is uniformly Abel ergodic.

The condition (i) holds whenever $\omega_{0} \leq 0$, let us also mention that somewhat different necessary and sufficient 
conditions are obtained in [14]. Clearly, if $T(t)$ is uniformly Cesàro ergodic then is uniformly Abel ergodic, but the reverse is not true, for more information see [9, Chapter 2]. It is useful to mention that the limit of Cesàro averages and of Abel averages of $C_{0}$-semigroup $\{T(t)\}_{t \geqslant 0}$ is the same, is the projection $P$ of $\mathcal{X}$ onto $\mathcal{N}(A)$ parallel to $\mathcal{R}(A)$, corresponding to the ergodic decomposition

$$
X=\mathcal{R}(A) \oplus \mathcal{N}(A) .
$$

The classical uniform ergodic theorem for $C_{0}$-semigroups $\{T(t)\}_{t \geq 0}$ of bounded linear operators on $X$, goes back to $\mathrm{M}$. Lin in [10], he treats the Cesàro ergodicity of a $C_{0}$-semigroup $\{T(t)\}_{t \geq 0}$ under the assumption $\lim _{t \rightarrow \infty}\|T(t)\| / t=0$, it showed that $T(t)$ is uniformly Cesàro ergodic if and only if its infinitesimal generator $A$ has a closed range if and only if $T(t)$ is uniformly Abel ergodic. In this case, and under this latter assumption, we can easy checked that $T(t)$ is uniformly Abel ergodic if and only if $\mathcal{X}=\mathcal{R}(A) \oplus \mathcal{N}(A)$. Moreover, this theory also plays an important role in the study of power convergence of linear operators. Recall that, an operator $T \in$ $\mathcal{B}(X)$ is called uniformly power convergent if there exists an operator $P \in \mathcal{B}(X)$ such that $\lim _{n \rightarrow \infty}\left\|T^{n}-P\right\|=0$. Lin, Shoikhet and Suciu in [11], showed that for a $C_{0}$-semigroup $\{T(t)\}_{t \geq 0}$ on $\mathcal{B}(X)$ satisfying $\lim _{t \rightarrow \infty}\|T(t)\| / t=$ $0,\{T(t)\}_{t \geq 0}$ is uniformly ergodic if and only if there exists some $\lambda>0$ such that the Abel average $\lambda R(\lambda, A)$ is uniformly power convergent. Kozitsky, Shoikhet and Zemànek in [8], obtained necessary and sufficient conditions for which the Abel averages of a $C_{0}$-semigroup $T(t)$ can be uniformly power convergent. Further condition have been obtained more recently by several authors $[8,16]$.

This paper is organized as follows. In section 2, we give some definitions and fundamental properties for an integrated semigroup of bounded linear operators on $X$. In section 3, we are motivated by application to the ergodic theory for an integrated semigroup $\{S(t)\}_{t \geq 0}$ on $\mathcal{B}(X)$. More precisely, We show that $S(t)$ is uniformly Abel ergodic if and only if $X=\mathcal{R}(A) \oplus \mathcal{N}(A)$, if and only if $\mathcal{R}\left(A^{k}\right)$ is closed for some integer $k$ and $\left\|\lambda^{2} R(\lambda, A)\right\| \longrightarrow 0$ as $\lambda \rightarrow 0^{+}$. Also, we show that if $S(t)$ satisfying $S(t) / t^{2} \longrightarrow 0$ as $t \rightarrow \infty$, then we have the following equivalent

(i) $S(t)$ is uniformly Cesàro ergodic,

(ii) $S(t)$ is uniformly Abel ergodic,

(iii) $\mathcal{R}\left(A^{k}\right)$ is closed for some integer $k \geq 1$,

(iv) The descent $\operatorname{des}(A)$ of $A$ is finite.

\section{Preliminaries}

We start this present section by recalling an interesting concept in operator theory that we need in the sequel. Let $A$ be a closed linear operator on a Banach space $X$, with domain $D(A) \subset X$. The smallest nonnegative integer $p$ such that $\mathcal{N}\left(A^{p}\right)=\mathcal{N}\left(A^{p+1}\right)$ is called the ascent of $A$ and denoted by $\operatorname{asc}(A)$. If such an integer does not exist, we set $\operatorname{asc}(A)=\infty$. Similarly, the smallest non-negative integer $q$ such that $\mathcal{R}\left(A^{q}\right)=$ $\mathcal{R}\left(A^{q+1}\right)$ is called the descent of $A$ and denoted by $\operatorname{des}(A)$. If such an integer does not exist, we set $\operatorname{des}(A)=\infty$. Let $A$ be a closed linear operator with $D(A) \subsetneq \mathcal{X}$, if $\operatorname{asc}(A)$ and $\operatorname{des}(A)$ are both finite, then $\operatorname{asc}(A) \leq \operatorname{des}(A)$, the equality holds when $A \in \mathcal{B}(X)$, see [18, Theorem 6.2]).

For $A \in \mathcal{B}(X)$, we have the following equivalences, see [4, Lemma 1.1]:

$$
\begin{aligned}
& \operatorname{asc}(A) \leq p \Longleftrightarrow \mathcal{R}\left(A^{p}\right) \cap \mathcal{N}\left(A^{j}\right)=\{0\} ; j=1,2, \ldots . \\
& \operatorname{des}(A) \leq q \Longleftrightarrow \mathcal{X}=\mathcal{R}\left(A^{j}\right)+\mathcal{N}\left(A^{q}\right) ; j=1,2, \ldots .
\end{aligned}
$$

The family $\{S(t)\}_{t \geqslant 0}$ of bounded linear operator on $\mathcal{B}(X)$ is called integrated semigroup [7, Definition 1.1] if it has the following properties:

1. $S(0)=0$, 
2. $S(s) S(t)=\int_{0}^{s} S(r+t)-S(r) d r$ for $t, s \geq 0$,

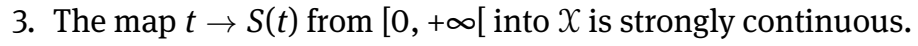

The differentiation spaces $C^{n}, n \geq 0$, are defined by $C^{0}=X$ and

$$
C^{n}=\left\{x \in X: S(.) x \in C^{n}\left(\mathbb{R}^{+} ; X\right)\right\} .
$$

Using this notion (2) can equivalently be formulated by $S(t) x \in C^{1}$ for all $x \in X$, and

$$
S^{\prime}(r) S(t)=S(r+t)-S(r)
$$

The set $N=\{x \in X ; S(t) x=0, \forall t \geq 0\}$ is called the degeneration space of the integrated semigroup $\{S(t)\}_{t \geqslant 0}$. In this case, $\{S(t)\}_{t \geqslant 0}$ is called non-degenerate if $N=\{0\}$ and degenerate otherwise.

The generator $A: D(A) \subset \mathcal{X} \rightarrow \mathcal{X}$ of a non-degenerate integrated semigroup $\{S(t)\}_{t \geqslant 0}$ is defined as follows: $x \in D(A)$ and $A x=y$ if and only if $x \in C^{1}$ and $S^{\prime}(t) x-x=S(t) y$ for $t \geq 0$. Usually, a non-degenerate integrated semigroup is uniquely determined by its generator. Motivated from the Laplace transform theory we can define the generator of an integrated semigroup as

$$
A x=\left(\lambda-R_{\lambda}^{-1}\right) \text { for all } x \in D(A),
$$

where $R_{\lambda}=\lambda \int_{0}^{+\infty} e^{-\lambda t} S(t) d t$ is the The Laplace Transformation of $\{S(t)\}_{t \geqslant 0}$, this integral does not exist in general, for more details see [7, Exemple 1.2]. To ensure the existence of this integral, we recall the following definition. An integrated semigroup $\{S(t)\}_{t \geqslant 0}$ is called exponentially bounded, if there exist constants $M>0$ and $\omega \in \mathbb{R}$ such that

$$
\|S(t)\| \leqslant M e^{\omega t} \text { for all } t \geqslant 0 \text {. }
$$

In this case, the Laplace Transformation $R_{\lambda}$ exists for all $\lambda$ with $\operatorname{Re} \lambda>\omega$. In General, an operator $A$ is called generator of an integrated semigroup, if there exists $\omega \in \mathbb{R}$ such that $(\omega, \infty) \subset \rho(A)$, and there exists a strongly continuous exponentially bounded family $\{S(t)\}_{t \geqslant 0}$ of bounded linear operators on $X$ such that

$$
\begin{aligned}
S(0) & =0, \text { and } \\
(\lambda-A)^{-1} & =\lambda \int_{0}^{\infty} e^{-\lambda t} S(t) d t, \text { for all } \lambda \text { with } \operatorname{Re} \lambda>\omega .
\end{aligned}
$$

In this case, the Laplace Transformation $R_{\lambda}$ of $\{S(t)\}_{t \geqslant 0}$ is exactly the resolvent function of $A$ and satisfies the pseudo-resolvent:

$$
R(\lambda)-R(\mu)=(\mu-\lambda) R(\lambda) R(\mu)
$$

For more details, we refer the interested reader to $[1,5,7]$.

Example 1. 1. Let $X=C[0,1]$ with $A f=f^{\prime}$ on $D(A)=\left\{f \in C^{1}([0,1]): f(1)=0\right\}$. The integrated semigroup generated by $A$ is given by

$$
S(t) f(x)=\int_{x}^{m} f(s) d s, \text { where } m=\min \{1, x+t\}
$$

2. We consider $X=\ell^{2}$ and the family $\{S(t)\}_{t \geqslant 0}$ of bounded linear operators on $X$ defined by:

$$
S(t)\left(x_{n}\right)_{n \in \mathbb{N}^{*}}=\left(\int_{0}^{t} e^{a_{n} s} d s x_{n}\right)_{n \in \mathbb{N}^{*}},
$$


where $a_{n}=n+2^{n^{2}} \pi i$. Then $\{S(t)\}_{t \geqslant 0}$ is an integrated semigroup on $X$.

3. Consider the Schrödinger operator $A=i . \Delta$ on $L^{p}(\mathbb{R})$ for $p \geq 1$. Then A generates an integrated semigroup $\left(\{S(t)\}_{t \geq 0}\right.$ given by

$$
f \mapsto \mathcal{F}^{-1}\left(u_{t} \mathcal{F} f\right) \text { with } u_{t}(\xi)=\int_{0}^{t} e^{-i s|\xi|^{2}} d s
$$

Definition 2.1. Let $\{S(t)\}_{t \geqslant 0}$ be an integrated semigroup on $\mathcal{B}(X)$.

(i) We say that $\{S(t)\}_{t \geqslant 0}$ is uniformly Cesàro ergodic if the Cesàro averages of $\{S(t)\}_{t \geq 0}$ defined by

$$
\mathcal{C}(t)=\frac{1}{t^{2}} \int_{0}^{t} S(r) d r ; \text { for } t \geq 0,
$$

converges in the norm operator topology as $t$ tend to infinity. Moreover, $\{S(t)\}_{t \geqslant 0}$ is said to be uniformly Cesàro bounded if there exists $M>0$ such that

$$
\sup _{t \geq 0}\|\mathcal{C}(t)\| \leq M .
$$

(ii) We say that $\{S(t)\}_{t \geqslant 0}$ is uniformly Abel ergodic if the Abel averages of $\{S(t)\}_{t \geqslant 0}$ defined by

$$
\mathcal{A}(\lambda)=\lambda^{2} \int_{0}^{\infty} e^{-\lambda t} S(t) d t ; \text { for } t \geq 0,
$$

converges in the norm operator topology as $\lambda$ tend to zero.

Now, we will need the following relations between integrated semigroups and their generators. The first result was investigated by W. Arendt [1] in the case of $n$-times integrated semigroup on $\mathcal{B}(X)$, where $n \in \mathbb{N}$.

Proposition 2.1. [1, Proposition 3.3] Let $A$ be the generator of an integrated semigroup $\{S(t)\}_{t \geqslant 0}$ on $\mathcal{B}(X)$. Then

1. For all $x \in D(A)$ and all $t \geq 0$, we have: $S(t) x \in D(A)$ and $A S(t) x=S(t) A x$.

Moreover, $S(t) x=\int_{0}^{t} S(s) A x d s+t x$.

2. For all $x \in X, \int_{0}^{t} S(s) x d s \in D(A)$, and

$$
A \int_{0}^{t} S(s) x d s=S(t) x-t x
$$

Lemma 2.1. [15, Lemma 2.3] Let $A$ be the generator of an integrated semigroup $\{S(t)\}_{t \geqslant 0}$ on $\mathcal{B}(X)$. Then we have the following assertions:

1. $\mathcal{R}(A)=(\lambda R(\lambda, A)-I) X$.

2. $\mathcal{N}(A)=\mathcal{N}(\lambda R(\lambda, A)-I)=\{x \in \mathcal{X}: S(t) x=t x$; for all $t \geq 0\}$.

We recall the following result which was recently proved in [16] for an $\alpha$-times integrated semigroup $\{S(t)\}_{t \geq 0}$ on $\mathcal{B}(X)$, where $\alpha \geq 0$.

Theorem 2.1. [16, Theorem 2.2] Let $A$ be the generator of an integrated semigroup $\{S(t)\}_{t \geqslant 0}$ on $\mathcal{B}(X)$ such that $\lim _{t \rightarrow \infty}\left\|\frac{S(t)}{t}\right\|=0$. If $\mathcal{R}(A)$ is closed, then $\{S(t)\}_{t \geq 0}$ is uniformly Cesàro ergodic. 


\section{Main results}

We began this section by the following two lemmas which will be widely used in the sequel.

Lemma 3.1. Let $A$ be the generator of an integrated semigroup $\{S(t)\}_{t \geqslant 0}$ on $\mathcal{B}(X)$. If $\left\|\lambda^{2} R(\lambda, A)\right\| \longrightarrow 0$ as $\lambda \rightarrow 0^{+}$, then $\overline{\mathcal{R}(A)} \cap \mathcal{N}(A)=\{0\}$, which yields asc $(A) \leq 1$.

Proof. Let $\{S(t)\}_{t \geqslant 0}$ be an integrated semigroup on $\mathcal{B}(X)$ with $A$ be their generator and $R(\lambda, A)$ the resolvent function of $A$. We assume that $\left\|\lambda^{2} R(\lambda, A)\right\| \rightarrow 0$ as $\lambda \rightarrow 0^{+}$and let $y \in \overline{\mathcal{R}(A)} \cap \mathcal{N}(A)$, then by the second assertion of Lemma 2.1, we get

$$
\lambda R(\lambda, A) y=y, \text { for all } \lambda \in \rho(A) .
$$

Since $\overline{\mathcal{R}(A)}=\overline{\mathcal{R}(\lambda R(\lambda, A)-I)}$, then there exists $x \in \mathcal{X}$ and $M>0$, such that

$$
y=(\lambda R(\lambda, A)-I) x \text { and }\|x\| \leq M\|y\| .
$$

Next, from the resolvent equation:

$$
R(\lambda, A)-R(\mu, A)=(\mu-\lambda) R(\lambda, A) R(\mu, A), \text { for all } \lambda \neq \mu \in \rho(A) .
$$

We get the following inequality, for all $\lambda \neq \mu$,

$$
\begin{aligned}
\|\lambda R(\lambda, A) y\| & \leq|\mu-\lambda|^{-1}\left[\left\|\lambda^{2} R(\lambda, A)\right\|+|\lambda|\|\mu R(\mu, A)\|\right]\|x\| \\
& \leq M|\mu-\lambda|^{-1}\left[\left\|\lambda^{2} R(\lambda, A)\right\|+|\lambda|\|\mu R(\mu, A)\|\right]\|y\|,
\end{aligned}
$$

It follows that $\lambda R(\lambda, A) y \longrightarrow 0$ as $\lambda \rightarrow 0^{+}$, which yields that $y=0$. Therefore $\overline{\mathcal{R}(A)} \cap \mathcal{N}(A)=\{0\}$.

Lemma 3.2. Let $A$ be a closed linear operator with domain $D(A) \subset X$, such that asc $(A)=d<\infty$. If either of the following hold:

(i) $\mathcal{R}\left(A^{n}\right)$ is closed for some $n>d$, or

(ii) $\mathcal{R}\left(A^{j}\right)+\mathcal{N}\left(A^{k}\right)$ is closed for some positive integers with $j+k=n \geq d$, then $\mathcal{R}\left(A^{n}\right)$ is closed for all $n \geq d$, and $\mathcal{R}\left(A^{j}\right)+\mathcal{N}\left(A^{k}\right)$ is closed for all $j+k \geq d$.

Proof. Let $A$ and $B$ are closed linear operators on a Banach space $\mathcal{X}$, if $\mathcal{R}(B) \subset D(A)$ then we have the following identity:

$$
A^{-1} \mathcal{R}(A B)=\mathcal{R}(B)+\mathcal{N}(A) .
$$

Then, for a linear operator $A$ on a Banach space $X$ and for some integers $j$ and $k$, we get

$$
A^{-k} \mathcal{R}\left(A^{j} A^{k}\right)=\mathcal{R}\left(A^{j}\right)+\mathcal{N}\left(A^{k}\right) .
$$

Then, we obtain the following results:

(i) If $\mathcal{R}\left(A^{n}\right)$ is closed, so is $\mathcal{R}\left(A^{j}\right)+\mathcal{N}\left(A^{k}\right)$ whenever $j+k=n$.

(ii) For $n \geq d, \mathcal{R}\left(A^{n}\right)$ is closed whenever $\mathcal{R}\left(A^{n}\right)+\mathcal{N}\left(A^{m}\right)$ is closed for some $m \geq 1$.

Assume that $A$ be a closed linear operator with domain $D(A) \subset X$ such that $\operatorname{asc}(A)=d<\infty$, then

$$
\mathcal{R}\left(A^{d}\right) \cap \mathcal{N}\left(A^{m}\right)=\{0\}, \text { for all } m=1,2, \ldots
$$

Now, we separate the hypothesis. Let $\mathcal{R}\left(A^{n}\right)$ is closed for some $n>d$, hence it follows from $(i)$ that $\mathcal{R}\left(A^{j}\right)+$ $\mathcal{N}\left(A^{k}\right)$ is closed whenever $j+k=n$. So take $j=n-1$ and $k=1$, then by $(i i), \mathcal{R}\left(A^{n-1}\right)$ is closed. Therefore, by induction $\mathcal{R}\left(A^{j}\right)$ is closed for all $d \leq j \leq n$. Since the ascent $\operatorname{asc}(A)$ is finite which means that $\mathcal{R}\left(A^{n-1}\right) \cap \mathcal{N}(A)=$ $\{0\}$, then the restriction of $A$ to the closed invariant subspace $\mathcal{R}\left(A^{n-1}\right)$ is one to one. Thus the restriction is a Banach space isomorphism from the closed subspace $\mathcal{R}\left(A^{n-1}\right)$ onto the closed subspace $\mathcal{R}\left(A^{n}\right)$. It carries the subspace $\mathcal{R}\left(A^{n}\right)$ onto $\mathcal{R}\left(A^{n+1}\right)$, which must be also closed. Consequently $\mathcal{R}\left(A^{n}\right)$ is closed for all $n \geq d$.

Next, we assume that $\mathcal{R}\left(A^{n}\right)+\mathcal{N}\left(A^{m}\right)$ is closed for some $n>d$ and $m \geq 1$. It follows from (ii) that $\mathcal{R}\left(A^{n}\right)$ is closed for some $n>d$. Again, we applied the first argument, we deduce $\mathcal{R}\left(A^{n}\right)$ is closed for all $n \geq d$, so is $\mathcal{R}\left(A^{n}\right)+\mathcal{N}\left(A^{m}\right)$ for all $n \geq d$ and $m \geq 1$. 
The first main result of this paper can be described as follows.

Theorem 3.1. Let $A$ be the generator of an integrated semigroup $\{S(t)\}_{t \geqslant 0}$ on $\mathcal{B}(X)$. Then the following assertions are equivalent:

1. $S(t)$ is uniform Abel ergodic,

2. $X=\mathcal{R}(A) \oplus \mathcal{N}(A)$,

3. $\left\|\lambda^{2} R(\lambda, A)\right\| \longrightarrow 0$ as $\lambda \rightarrow 0^{+}$and $\mathcal{R}(A)$ is closed,

4. $\left\|\lambda^{2} R(\lambda, A)\right\| \longrightarrow 0$ as $\lambda \rightarrow 0^{+}$and the descent des $(A)$ is finite.

Proof. Let $A$ be the generator of an integrated semigroup $\{S(t)\}_{t \geqslant 0}$ on $\mathcal{B}(X)$. then there exist constants $M>0$ and $\omega \in \mathbb{R}$ such that

$$
\|S(t)\| \leqslant M e^{\omega t} \text { for all } t \geqslant 0 .
$$

Therefore, the Laplace Transformation $R_{\lambda}$ of $\{S(t)\}_{t \geqslant 0}$ is exactly the resolvent of $A$. It follows that the Abel averages $\mathcal{A}(\lambda)$ of $\{S(t)\}_{t \geqslant 0}$ satisfying the following equality

$$
\begin{aligned}
\lim _{\lambda \rightarrow 0^{+}} \mathcal{A}(\lambda) & =\lim _{\lambda \rightarrow 0^{+}} \lambda^{2} \int_{0}^{\infty} e^{-\lambda t} S(t) d t ; \text { for } t \geq 0, \\
& =\lim _{\lambda \rightarrow 0^{+}} \lambda R(\lambda, A) .
\end{aligned}
$$

$(1) \Rightarrow$ (2) It is known from the mean ergodic theorem [19, p. 217] that if there exists an operator $P \in \mathcal{B}(X)$ such that $\|\lambda R(\lambda, A)-P\| \longrightarrow 0$ as $\lambda \rightarrow 0^{+}$, then $P$ is the projection of $X$ onto $\mathcal{N}(\lambda R(\lambda, A)-I)$ parallel to $(\lambda R(\lambda, A)-I) X$, and by Lemma 2.1 , we get

$$
X=\mathcal{R}(A) \oplus \mathcal{N}(A) .
$$

(2) $\Longrightarrow$ (3) Assume that $X=\mathcal{R}(A) \oplus \mathcal{N}(A)$ where $\mathcal{R}(A)$ is closed. It follows from the second assertion of Lemma 2.1 that for all $x \in \mathcal{N}(A)$ we have $\lambda R(\lambda, A) x=x$. Then

$$
\left\|\left.\lambda^{2} R(\lambda, A)\right|_{\mathcal{N}(A)}\right\| \longrightarrow 0 \text { as } \lambda \rightarrow 0^{+} .
$$

So, to complete the proof we show that $\left\|\left.\lambda^{2} R(\lambda, A)\right|_{\mathcal{R}(A)}\right\| \longrightarrow 0$ as $\lambda \rightarrow 0^{+}$. Indeed, let's denote $Y=\mathcal{R}(A)$ and $A_{1}$ be the generator of the restriction of $T(t)$ to $Y$, which is equal to the restriction of $A$ to $Y \cap D(A)$. From the first assertion of Lemma 2.1 we have $Y=\mathcal{R}(\lambda R(\lambda, A)-I)$. Since $\mathcal{R}(\lambda R(\lambda, A)-I) \cap \mathcal{N}(\lambda R(\lambda, A)-I)=\{0\}$, then the operator $(\lambda R(\lambda, A)-I)$ is invertible on $Y$.

Now, let $y \in Y \cap D(A)$ such that $A_{1} y=0$, hence

$$
\begin{aligned}
y & =R(\lambda, A)(\lambda-A) y \\
& =\lambda R(\lambda, A) y-R(\lambda, A) A y \\
& =\lambda R(\lambda, A) y-R(\lambda, A) A_{1} y \\
& =\lambda R(\lambda, A) y .
\end{aligned}
$$

Then $y \in \mathcal{N}(\lambda R(\lambda, A)-I)$, which implies that $y=0$. Thus $A_{1}$ is one to one.

Clearly, we have $R(\lambda, A) Y \subset Y$, hence we obtain that $(\lambda R(\lambda, A)-I) Y \subset \mathcal{R}\left(A_{1}\right)$.

Then, we get the following

$$
Y \supset \mathcal{R}\left(A_{1}\right) \supset(\lambda R(\lambda, A)-I) Y=(\lambda R(\lambda, A)-I) X=\mathcal{R}(A)=Y .
$$

Hence $Y=\mathcal{R}\left(A_{1}\right)$, so $A_{1}^{-1}$ is defined on all $Y$, since $A_{1}$ is closed, therefore $A_{1}^{-1}$ is closed, and by the closed graph theorem $A_{1}^{-1}$ is continuous.

Let $0<\lambda<\delta<\frac{1}{\left\|A_{1}^{-1}\right\|}$ and $y \in Y$, we get

$$
\left\|\lambda^{2} R(\lambda, A) y\right\|=\left\|\lambda^{2} R(\lambda, A) A_{1} A_{1}^{-1} y\right\|
$$




$$
\leq \quad\left\|\lambda^{2}(\lambda R(\lambda, A)-I)\right\|\left\|A_{1}^{-1}\right\|\|y\| .
$$

Hence

$$
\left\|\lambda^{2} R(\lambda, A) y\right\| \leq \lambda^{2}(\|\lambda R(\lambda, A)\|+1)\left\|A_{1}^{-1}\right\|\|y\| .
$$

Also, we have

$$
\|\lambda R(\lambda, A)\| \leq \delta(\|\lambda R(\lambda, A)\|+1)\left\|A_{1}^{-1}\right\| .
$$

Then, we get

$$
\|\lambda R(\lambda, A)\| \leq \frac{\delta\left\|A_{1}^{-1}\right\|}{1-\delta\left\|A_{1}^{-1}\right\|}=M .
$$

Therefore

$$
\begin{aligned}
\left\|\lambda^{2} R(\lambda, A) y\right\| & \leq\left\|\lambda^{2}(\lambda R(\lambda, A)-I)\right\|\left\|A_{1}^{-1}\right\|\|y\| \\
& \leq \lambda^{2}(\|\lambda R(\lambda, A)\|+1)\left\|A_{1}^{-1}\right\|\|y\| \\
& \leq \lambda^{2}(M+1)\left\|A_{1}^{-1}\right\|\|y\|,
\end{aligned}
$$

which implies that $\left\|\left.\lambda^{2} R(\lambda, A)\right|_{Y}\right\| \longrightarrow 0$ as $\lambda \rightarrow 0^{+}$. Hence the assertion (3) holds.

(3) $\Longrightarrow$ (4) suppose that $\left\|\lambda^{2} R(\lambda, A)\right\| \longrightarrow 0$ as $\lambda \rightarrow 0^{+}$, and $\mathcal{R}(A)$ is closed.

By Lemma 2.1, we have $\mathcal{R}(A)=(\lambda R(\lambda, A)-I) X$, which means that for all $\lambda>0$, the operator $\lambda R(\lambda, A)-I$ has a closed range. Fix $\mu>0$ such that for each $y \in(\mu R(\mu, A)-I) X$, there exists a $M>0$ and $x \in X$ such that $y=(\mu R(\mu, A)-I) x$ and $\|x\| \leq M\|y\|$. So, we have

$$
\lambda R(\lambda, A)(\mu R(\mu, A)-I)=\lambda \mu R(\lambda, A) R(\mu, A)-\lambda R(\lambda, A) .
$$

By the resolvent equation:

$$
R(\lambda, A)-R(\mu, A)=(\mu-\lambda) R(\lambda, A) R(\mu, A),
$$

we obtain

$$
\begin{aligned}
\lambda R(\lambda, A)(\mu R(\mu, A)-I) & =\lambda \mu R(\lambda, A) R(\mu, A)-\lambda(\mu-\lambda) R(\lambda, A) R(\mu, A)-\lambda R(\mu, A) \\
& =\lambda^{2} R(\lambda, A) R(\mu, A)-\lambda R(\mu, A) \\
& =\lambda^{2}(\mu-\lambda)^{-1}[R(\lambda, A)-R(\mu, A)]-\lambda R(\mu, A) \\
& =(\mu-\lambda)^{-1}\left[\lambda^{2} R(\lambda, A)-\lambda \mu R(\mu, A)\right] .
\end{aligned}
$$

This gives

$$
\begin{aligned}
\|\lambda R(\lambda, A) y\| & =\|\lambda R(\lambda, A)(\mu R(\mu, A)-I) x\| \\
& =\left\|(\mu-\lambda)^{-1}\left[\lambda^{2} R(\lambda, A)-\lambda \mu R(\mu, A)\right] x\right\| \\
& \leq|\mu-\lambda|^{-1}\left[\left\|\lambda^{2} R(\lambda, A)\right\|+|\lambda|\|\mu R(\mu, A)\|\right] M\|y\| .
\end{aligned}
$$

It follows that

$$
\left\|\left.\lambda R(\lambda, A)\right|_{(\lambda R(\lambda, A)-I) x}\right\| \longrightarrow 0 \text { as } \lambda \rightarrow 0^{+} .
$$

Then for a small $\lambda>0$, the operator $\lambda R(\lambda, A)-I$ is invertible on $(\lambda R(\lambda, A)-I) x$. Therefore

$$
(\lambda R(\lambda, A)-I)^{2} X=(\lambda R(\lambda, A)-I) X,
$$

which yields $X=(\lambda R(\lambda, A)-I) X+\mathcal{N}(\lambda R(\lambda, A)-I)$. It follows from Lemma 2.1 that $X=\mathcal{R}(A)+\mathcal{N}(A)$, therefore the $\operatorname{des}(A)$ is finite. Hence the assertion (4) holds.

(4) $\Rightarrow$ (1) Since the $\operatorname{des}(A)$ is finite then $\mathcal{X}=\mathcal{R}(A)+\mathcal{N}\left(A^{d}\right)$ for some integer $d>0$, and by Lemma 3.1, we have $\overline{\mathcal{R}(A)} \cap \mathcal{N}(A)=\{0\}$, then $\mathcal{X}=\mathcal{R}(A) \oplus \mathcal{N}(A)$. Hence, it follows from Lemma 2.1

$$
X=(\lambda R(\lambda, A)-I) X \oplus \mathcal{N}(\lambda R(\lambda, A)-I) .
$$

Moreover, as shown above that $\left\|\left.\lambda R(\lambda, A)\right|_{(\lambda R(\lambda, A)-I) x}\right\| \longrightarrow 0$ as $\lambda \rightarrow 0^{+}$. Since for all $x \in \mathcal{N}(\lambda R(\lambda, A)-I)$, we have $\lambda R(\lambda, A) x=x$, then $\left.\lambda R(\lambda, A)\right|_{\mathcal{N}(\lambda R(\lambda, A)-I)}$ converge to the identity operator $I$ as $\lambda \rightarrow 0^{+}$. It follows from the decomposition (3.2) that $\lambda R(\lambda, A)$ converges uniformly on $X$. Hence the assertion (1) holds. 
The following corollary is an immediate consequence of the Theorem 3.2 and of the Lemma 3.2.

Corollary 3.1. Let $A$ be the generator of an integrated semigroup $\{S(t)\}_{t \geqslant 0}$ on $\mathcal{B}(X)$. Then the following assertions are equivalent:

1. $S(t)$ is uniform Abel ergodic,

2. $\left\|\lambda^{2} R(\lambda, A)\right\| \longrightarrow 0$ as $\lambda \rightarrow 0^{+}$and $\mathcal{R}\left(A^{k}\right)$ is closed for some integer $k$,

3. $\left\|\lambda^{2} R(\lambda, A)\right\| \longrightarrow 0$ as $\lambda \rightarrow 0^{+}$and $\mathcal{R}\left(A^{k}\right)+\mathcal{N}\left(A^{j}\right)$ is closed for some $k, j>0$.

Corollary 3.2. Let $A$ be the generator of an integrated semigroup $\{S(t)\}_{t \geqslant 0}$ on $\mathcal{B}(X)$. Then the following assertions are equivalent:

1. $S(t)$ is uniformly Abel ergodic,

2. $\mathcal{R}(A)$ is closed and $\left\|\left.\lambda R(\lambda, A)\right|_{\mathcal{R}(A)}\right\| \longrightarrow 0$ as $\lambda \rightarrow 0^{+}$.

Proof. Let $A$ be the generator of an integrated semigroup $\{S(t)\}_{t \geqslant 0}$ on $\mathcal{B}(X)$ and let $\left.R(\lambda, A)\right|_{\mathcal{R}(A)}$ be the restriction of $R(\lambda, A)$ onto $\mathcal{R}(A)$. As shown above in the proof of Theorem 3.2 that if $S(t)$ is uniformly Abel ergodic, then $\mathcal{R}(A)$ is closed and $\left\|\left.\lambda R(\lambda, A)\right|_{\mathcal{R}(A)}\right\| \longrightarrow 0$ as $\lambda \rightarrow 0^{+}$by (3.1).

Conversely, we assume that $\mathcal{R}(A)$ is closed and $\left\|\left.\lambda R(\lambda, A)\right|_{\mathcal{R}(A)}\right\| \longrightarrow 0$ as $\lambda \rightarrow 0^{+}$.

Since $\mathcal{R}(A)=(\lambda R(\lambda, A)-I) X$, then $\left\|\left.\lambda R(\lambda, A)\right|_{(\lambda R(\lambda, A)-I) X}\right\| \longrightarrow 0$ as $\lambda \rightarrow 0^{+}$, then for a small $\lambda$, the operator $\left.(\lambda R(\lambda, A)-I)\right|_{(\lambda R(\lambda, A)-I) X}$ is invertible and we have

$$
\mathcal{R}(\lambda R(\lambda, A)-I)=\mathcal{R}\left(\left.(\lambda R(\lambda, A)-I)\right|_{\mathcal{R}(A)}\right)=\mathcal{R}\left[(\lambda R(\lambda, A)-I)^{2}\right] .
$$

Therefore

$$
X=(\lambda R(\lambda, A)-I) X+\mathcal{N}(\lambda R(\lambda, A)-I) .
$$

Now, let $y \in \mathcal{R}(\lambda R(\lambda, A)-I) \cap \mathcal{N}(\lambda R(\lambda, A)-I)$, so $\lambda R(\lambda, A) y=y$ for all $\lambda>0$, and by assumption $\lambda R(\lambda, A) y \longrightarrow$ 0 as $\lambda \rightarrow 0^{+}$, hence $y=0$ which yields that

$$
(\lambda R(\lambda, A)-I) X \cap \mathcal{N}(\lambda R(\lambda, A)-I)=\{0\} .
$$

Then, the summation in (3.3) is direct and from Lemma $2.1 \mathcal{X}=\mathcal{R}(A) \oplus \mathcal{N}(A)$. Finally, Theorem 3.2 implies that $S(t)$ is uniformly Abel ergodic.

Now, we present our second main result as follows.

Theorem 3.2. Let $A$ be the generator of an integrated semigroup $\{S(t)\}_{t \geqslant 0}$ on $\mathcal{B}(\mathcal{X})$. Assume that $\lim _{t \rightarrow \infty}\|S(t)\| / t^{2}=0$, then $S(t)$ is uniformly Abel ergodic if and only if $S(t)$ is uniformly Cesàro ergodic.

Proof. Let $A$ be the generator of an integrated semigroup $\{S(t)\}_{t \geqslant 0}$ on $\mathcal{B}(X)$ such that $\lim _{t \rightarrow \infty}\|S(t)\| / t^{2}=0$. Let $S(t)$ be uniformly Abel ergodic, then by Theorem 3.1, we obtain the decomposition $X=\mathcal{R}(A) \oplus \mathcal{N}(A)$, with $\mathcal{R}(A)$ is closed.

From the second assertion of Lemma 2.1, we can easy check that for all $x \in \mathcal{N}(A)$.

$$
\lim _{t \rightarrow \infty}\left\|\frac{1}{t^{2}} \int_{0}^{t} S(r) x d r-\frac{I x}{2}\right\|=0
$$

So, to complete the proof we show that $\frac{1}{t^{2}} \int_{0}^{t} S(r) y d r \longrightarrow 0$ as $t \rightarrow \infty$, for all $y \in \mathcal{R}(A)$. Since $\mathcal{R}(A)$ is closed, let's denote $A_{1}$ be the generator of the restriction of $S(t)$ to $\mathcal{R}(A)$, which is equal to the restriction of $A$ to $\mathcal{R}(A) \cap D(A)$. As shown in the proof of Theorem 3.1 that $A_{1}^{-1}$ is defined on all $\mathcal{R}(A)$ and continuous, then for all 
$y \in \mathcal{R}(A)$, there exists $x \in D(A)$ such that $y=A_{1} x$ and $\|x\| \leq\left\|A_{1}^{-1}\right\|\|y\|$. the second assertion of Proposition 2.1 implies that for all $x \in D(A)$, we have

$$
\int_{0}^{t} S(s) A x d s=S(t) x-t x ; \text { for all } t \geq 0
$$

It follows that, we get

$$
\begin{aligned}
\left\|\frac{1}{t^{2}} \int_{0}^{t} S(r) y d r\right\| & =\left\|\frac{1}{t^{2}}(S(t) x-t x)\right\| \\
& \leq\left\|A_{1}^{-1}\right\|\left(\left\|\frac{S(t)}{t^{2}}\right\|+\left|\frac{1}{t}\right|\right)\|y\| .
\end{aligned}
$$

Since $\lim _{t \rightarrow \infty}\|S(t)\| / t^{2}=0$, then $\left\|\frac{1}{t^{2}} \int_{0}^{t} S(r) y d r\right\| \longrightarrow 0$ as $t \rightarrow \infty$ for all $y \in \mathcal{R}(A)$. Therefore $S(t)$ is uniformly Cesàro ergodic.

Conversely, let $S(t)$ be uniformly Cesàro ergodic, hence there exists an operator $P \in \mathcal{B}(X)$ such that $\| \mathcal{C}(t)$ $P \| \longrightarrow 0$ as $t \rightarrow \infty$, where $\mathrm{C}(t)=\frac{1}{t^{2}} \int_{0}^{t} S(r) d r$ for $t \geq 0$. So, there exists $\varepsilon>0$ and $a>0$ such that $\|\mathcal{C}(t)-P\| \leq \varepsilon$ for all $t>a$.

Now, let's denote $\mathcal{W}(t)=\int_{0}^{t} S(r) d r$ for all $t \geq 0$. Then, using the integration by parts, we get the following identity:

$$
R(\lambda, A)=\lambda^{2} \int_{0}^{\infty} e^{-\lambda t} \mathcal{W}(t) d t, \text { for all } t \geq 0
$$

Moreover, we have the following identity for any bounded linear operator $P \in \mathcal{B}(X)$

$$
\lambda^{\alpha+1} \int_{0}^{\infty} e^{-\lambda t} t^{\alpha} P d t=(\alpha !) P, \text { for all } \lambda \in \mathbb{C} \text { and } \alpha, t \geq 0 .
$$

It follows from (3.4) and (3.5), we have for every $x \in X$

$$
\begin{aligned}
\|\lambda R(\lambda, A) x-2 P x\| & =\left\|\lambda R(\lambda, A) x-\lambda^{3} \int_{0}^{\infty} e^{-\lambda t} t^{2} P x d t\right\| \\
& =\left\|\lambda^{3} \int_{0}^{\infty} e^{-\lambda t} \mathcal{W}(t) x d t-\lambda^{3} \int_{0}^{\infty} e^{-\lambda t} t^{2} P x d t\right\| \\
& \leq\left\|\lambda^{3} \int_{0}^{\infty} e^{-\lambda t}\left(\mathcal{W}(t)-\left(t^{2}\right) P\right) d t\right\|\|x\| \\
& \leq\left[\left|\lambda^{3}\right| \int_{0}^{a} e^{-\lambda t}\left(\|\mathcal{W}(t)\|+t^{2}\|P\|\right) d t\right. \\
& \leq\left[\left|\lambda^{3}\right| \int_{0}^{a} e^{-\lambda t}\left(\|\mathcal{W}(t)\|+t^{2}\|P\|\right) d t\right.
\end{aligned}
$$




$$
\begin{gathered}
\left.+\left\|\lambda^{3} \int_{a}^{\infty} e^{-\lambda t} t^{2}(\mathcal{C}(t)-P) d t\right\|\right]\|x\| \\
\leq\left[\left|\lambda^{3}\right| \int_{0}^{a} e^{-\lambda t}\left(\|\mathcal{W}(t)\|+t^{2}\|P\|\right) d t+2\|\mathcal{C}(t)-P\|\right]\|x\| \\
\leq\left[\left|\lambda^{3}\right| a\left(\sup _{t \leq a}\|\mathcal{W}(t)\|+a^{2}\|P\|\right)+2 \varepsilon\right]\|x\| .
\end{gathered}
$$

Therefore the above estimate implies that $\|\lambda R(\lambda, A)-2 P\| \longrightarrow 0$ as $\lambda \rightarrow 0^{+}$, which yields that $S(t)$ is uniformly Abel ergodic and the proof is finished.

Corollary 3.3. Let $A$ be the generator of an integrated semigroup $\{S(t)\}_{t \geqslant 0}$ on $\mathcal{B}(X)$. Assume that $\lim _{t \rightarrow \infty}\|S(t)\| / t^{2}=0$, then the following assertions are equivalent:

1. $S(t)$ is uniformly Cesàro ergodic,

2. $\mathcal{R}\left(A^{k}\right)$ is closed for some integer $k \geq 1$,

3. $\mathcal{R}\left(A^{k}\right)+\mathcal{N}\left(A^{j}\right)$ is closed for some integers $k, j \geq 1$,

4. The descent $\operatorname{des}(A)$ of $A$ is finite.

Proof. Let $A$ be the generator of an integrated semigroup $\{S(t)\}_{t \geqslant 0}$ on $\mathcal{B}(X)$. First we must show that $\lim _{t \rightarrow \infty}\|S(t)\| / t^{2}=0$ implies that $\left\|\lambda^{2} R(\lambda, A)\right\| \longrightarrow 0$ as $\lambda \rightarrow 0^{+}$. Indeed, if $\lim _{t \rightarrow \infty}\|S(t)\| / t^{2}=0$, then there exists $\varepsilon>0$ and $a>0$ such that

$$
\|S(t)\| \leq \varepsilon t^{2} ; \text { for all } t>a
$$

Using the resolvent equation, we obtain for all $x \in X$ and $\mu \neq \lambda$;

$$
\begin{aligned}
\left\|\lambda^{2} R(\lambda, A) x\right\| & =\left\|\lambda^{2}[R(\mu, A)+(\mu-\lambda) R(\lambda, A) R(\mu, A)] x\right\| \\
& \leq\left\|\lambda^{2} R(\mu, A)\right\|\|x\|+|\mu-\lambda| \lambda^{2}\|R(\lambda, A) R(\mu, A) x\| \\
& \leq\left\|\lambda^{2} R(\mu, A)\right\|\|x\|+|\mu-\lambda| \lambda^{3} \int_{0}^{\infty} e^{-\lambda t}\|S(t) R(\mu, A) x\| d t \\
& \leq\left\|\lambda^{2} R(\mu, A)\right\|\|x\|+|\mu-\lambda|\left[\lambda^{3} \int_{0}^{a} e^{-\lambda t}\|S(t) R(\mu, A) x\| d t\right. \\
& \left.+\varepsilon \lambda^{3} \int_{a}^{\infty} e^{-\lambda t} t^{2}\|R(\mu, A) x\| d t\right] .
\end{aligned}
$$

From the identity (3.5), we obtain

$$
\begin{gathered}
\left\|\lambda^{2} R(\lambda, A) x\right\| \leq\left\|\lambda^{2} R(\mu, A)\right\|\|x\|+|\mu-\lambda|\left[\lambda^{3} a\left(\sup _{t \leq a}\|S(t)\|\|R(\mu, A)\|\right)\right. \\
+2 \varepsilon\|R(\mu, A)\|]\|x\| .
\end{gathered}
$$

It follows from the above estimate that $\left\|\lambda^{2} R(\lambda, A)\right\| \longrightarrow 0$ as $\lambda \rightarrow 0^{+}$. So, according to Corollary 3.1, Theorem 3.1 and Theorem 3.2, the equivalents hold.

Proposition 3.1. Let $A$ be the generator of an integrated semigroup $\{S(t)\}_{t \geqslant 0}$ on $\mathcal{B}(X)$. If $\{S(t)\}_{t \geqslant 0}$ is uniformly Cesàro ergodic, then the Laplace Transformation $R_{\lambda}$ of $\{S(t)\}_{t \geqslant 0}$ exists for all $\lambda$ with the real part $\operatorname{Re}(\lambda)>0$.

Proof. Assume that $\{S(t)\}_{t \geqslant 0} \subset \mathcal{B}(X)$ is uniformly Cesàro ergodic, then there exists $M>0$ such that

$$
\sup _{t \geq 0}\left\|\frac{1}{t^{2}} \int_{0}^{t} S(s) d s\right\| \leq M .
$$


Let's denote $\mathcal{W}(t)=\int_{0}^{t} S(s) d s$ for all $t \geq 0$, and let $\lambda \in \mathbb{C}$ such that $\operatorname{Re}(\lambda)>0$. Then, from the integration by parts, we have for all $0<u<v$ and $x \in X$

$$
\begin{aligned}
\left\|\lambda \int_{u}^{v} e^{-\lambda t} S(t) x d t\right\| & =\left\|\lambda\left[e^{-\lambda t} \mathcal{W}(t) x\right]_{u}^{v}+\lambda^{2} \int_{u}^{v} e^{-\lambda t} \mathcal{W}(t) x d t\right\| \\
& \leq M\left\|\lambda\left[e^{-\lambda t} t^{2}\right]_{u}^{v}+\lambda^{2} \int_{u}^{v} e^{-\lambda t} t^{2} d t\right\|\|x\| \\
& \leq M\left[|\lambda|\left(e^{-\lambda v} v^{2}+e^{-\lambda u} u^{2}\right)+\left|\lambda^{2}\right| \int_{u}^{v} e^{-R e \lambda t} t^{2} d t\right]\|x\| .
\end{aligned}
$$

It follows that $\left\|\lambda \int_{u}^{v} e^{-\lambda t} S(t) d t\right\| \longrightarrow 0$ as $u \rightarrow \infty$. Finally, we deduce that the Laplace Transformation $R_{\lambda}$ of $\{S(t)\}_{t \geqslant 0}$ exists for all $\lambda$ with $\operatorname{Re} \lambda>0$.

Proposition 3.2. Let $\{S(t)\}_{t \geqslant 0}$ be an integrated semigroup on $\mathcal{B}(X)$. Then, $S(t)$ is uniformly Cesàro ergodic if and only if the restriction of $S(t)$ to $\overline{\mathcal{R}(A)}$ is uniformly Cesàro ergodic.

Proof. Let $A$ be the generator of an integrated semigroup $\{S(t)\}_{t \geqslant 0}$ on $\mathcal{B}(X)$. Let's denote $\{Q(t)\}_{t \geqslant 0}$ be the restriction of $\{S(t)\}_{t \geqslant 0}$ to $\overline{\mathcal{R}(A)}$, and $A_{1}$ be their generator, which is exactly the restriction of $A$ to $\overline{\mathcal{R}(A)} \cap D(A)$. As shown above that if $S(t)$ is uniformly Cesàro ergodic, then the Cesàro averages of $S(t)$ converges uniformly to the projection $P$ of $X$ onto $\mathcal{N}(A)$ along $\mathcal{R}(A)$, corresponding to the ergodic decomposition $X=\mathcal{R}(A) \oplus \mathcal{N}(A)$ where $\mathcal{R}(P)=\mathcal{N}(A)$ and $\mathcal{N}(P)=\mathcal{R}(A)$. Since $\mathcal{R}(A)$ is closed and $S(t)$-invariant for all $t \geq 0$, then we easily check that $Q(t)$ is uniformly Cesàro ergodic to 0 , which means that the Cesàro averages of $Q(t)$ converge to 0 as $t \rightarrow \infty$.

Conversely, we assume that $Q(t)$ is uniformly Cesàro ergodic, so it follows from Theorem 3.2 that $Q(t)$ is also uniformly Abel ergodic and $\overline{\mathcal{R}(A)}=\mathcal{R}\left(A_{1}\right) \oplus \mathcal{N}\left(A_{1}\right)$ where $\mathcal{R}\left(A_{1}\right)$ is closed. Moreover, we have

$$
\left\|\frac{1}{t^{2}} \int_{0}^{t} S(r) x d r\right\| \rightarrow 0 \text { as } t \rightarrow \infty, \text { for all } x \in \overline{\mathcal{R}(A)} .
$$

Let $x \in \overline{\mathcal{R}(A)}$, hence from the above decomposition, we have $x=y+z$ where $y \in \mathcal{R}\left(A_{1}\right)$ and $z \in \mathcal{N}\left(A_{1}\right)$. Since $\mathcal{N}\left(A_{1}\right) \subset \mathcal{N}(A)$, then

$$
z=Q(t) z=\frac{1}{t^{2}} \int_{0}^{t} Q(r) z d r, \text { for all } t \geq 0
$$

Therefore, we obtain

$$
\begin{aligned}
\left\|\frac{1}{t^{2}} \int_{0}^{t} Q(r) z d r\right\| & =\left\|\frac{1}{t^{2}} \int_{0}^{t} \mathcal{Q}(r)(x-y) d r\right\| \\
& \leq\left\|\frac{1}{t^{2}} \int_{0}^{t} Q(r) x d u\right\|+\left\|\frac{1}{t^{2}} \int_{0}^{t} \mathcal{Q}(r) y d u\right\| .
\end{aligned}
$$

It follows that $\left\|\frac{1}{t^{2}} \int_{0}^{t} \mathcal{Q}(r) z d r\right\| \longrightarrow 0$ as $t \rightarrow \infty$, hence $z=0$ which gives that $x=y$. Therefore $\overline{\mathcal{R}(A)}=\mathcal{R}\left(A_{1}\right)$, since $\mathcal{R}\left(A_{1}\right) \subset \mathcal{R}(A)$, then $\mathcal{R}(A)$ is closed. 
Furthermore, since $Q(t)$ is uniformly Abel ergodic and their Cesàro averages converge to 0 , then

$$
\left\|\left.\lambda R(\lambda, A)\right|_{\mathcal{R}(A)}\right\| \longrightarrow 0 \text { as } \lambda \rightarrow 0^{+} .
$$

Consequently, $T(t)$ is uniformly Abel ergodic by Corollary 3.2, and we obtain

$$
X=\mathcal{R}(A) \oplus \mathcal{N}(A) .
$$

The projection $P$ of $X$ onto $\mathcal{N}(A)$ along $\mathcal{R}(A)$ corresponding to this decomposition is bounded, so we have $\mathcal{N}(I-P)=\mathcal{R}(P)=\mathcal{N}(A)$, and $\mathcal{R}(I-P)=\mathcal{N}(P)=\mathcal{R}(A)$. Then it follows from Lemma 2.1 that for all $x \in \mathcal{X}$

$$
\frac{1}{t^{2}} \int_{0}^{t} S(r) P x d r=\frac{1}{2} P x
$$

So, we obtain

$$
\begin{aligned}
\left\|\mathcal{C}(t) x-\frac{1}{2} P x\right\| & =\left\|\frac{1}{t^{2}} \int_{0}^{t} S(r) x d r-\frac{1}{t^{2}} \int_{0}^{t} S(r) P x d r\right\| \\
& =\left\|\frac{1}{t^{2}} \int_{0}^{t} S(r)(x-P x) d r\right\| \\
& =\left\|\frac{1}{t^{2}} \int_{0}^{t} Q(r)(x-P x) d r\right\| \\
& \leq\left\|\frac{1}{t^{2}} \int_{0}^{t} Q(r) d r\right\|\|x-P x\| \\
& \leq\left\|\frac{1}{t^{2}} \int_{0}^{t} Q(r) d r\right\|(1+\|P\|)\|x\| .
\end{aligned}
$$

Then $\left\|\mathcal{C}(t) x-\frac{1}{2} P x\right\| \longrightarrow 0$ as $t \rightarrow \infty$. Finally, $S(t)$ is uniformly Cesàro ergodic.

Remark 3.1. Let $A$ be the generator of an integrated semigroup $\{S(t)\}_{t \geqslant 0}$ on $\mathcal{B}(\mathcal{X})$. If $\lim _{t \rightarrow \infty}\|S(t)\| / t=0$, then $A$ is one to one by [15, Corollary 2.4]. In this case, if the Cesàro averages and the Abel averages converge, they will converge to zero and we obtain $X=\mathcal{R}(A)$. Moreover, the strong limit of $\frac{1}{t} \int_{0}^{t} S(r) d r$ may be divergent when $t \rightarrow \infty$, as the following example shows.

Example: Let $X=C([0, \infty])$, we consider the derivation operator $A f=-f^{\prime}$ for all $f \in D(A)$, with $D(A)=\{f \in$ $\left.C^{1}([0,1]): f(0)=0\right\}$. Since the domain $D(A)$ is not dense in $X$, then $A$ cannot be an infinitesimal generator of a $C_{0}$-semigroup. Furthermore, the semigroup $S(t)$ generated by $A$, is given by:

$$
(S(t) f)(x)=\left\{\begin{array}{ll}
-\int_{x}^{x-t} f(s) d s, & \text { si } x>t, \\
\int_{x}^{0} f(s) d s, & \text { si } 0 \leq x \leq t,
\end{array} .\right.
$$

Note that $S(t)$ is an integrated semigroup of type $\omega_{0}=0$, where

$$
\omega_{0}=\inf \left\{w \in \mathbb{R}: \text { there exists } M \text { such that }\|S(t)\| \leq M e^{w t}, t \geq 0\right\} .
$$


It follows that $\|S(t)\| / t \longrightarrow 0$ as $t \rightarrow \infty$.

On the other hand, since the generator $A$ has an empty spectrum, then $X=R(A)$, and by Theorem 3.1 and Corollary 3.3, we deduce that $S(t)$ is both uniformly Cesàro ergodic and uniformly Abel ergodic. More precisely, we have

$$
\lim _{t \rightarrow \infty}\left\|\frac{1}{t^{2}} \int_{0}^{t} S(r) d r\right\|=\lim _{\lambda \rightarrow 0^{+}}\left\|\lambda^{2} \int_{0}^{\infty} e^{-\lambda t} S(t) d t\right\|=0 .
$$

Now, we suppose that then there exists an operator $P$ such that

$$
\lim _{t \rightarrow \infty}\left\|\frac{1}{t} \int_{0}^{t} S(r) d r-P\right\|=0 .
$$

Hence $P$ is a projection operator of $X$ onto $\mathcal{N}(A)$ parallel to $\mathcal{R}(A)$, corresponding to ergodic decomposition $X=\mathcal{R}(A) \oplus \mathcal{N}(P)$. As shown above that $X=\mathcal{R}(A)$, then we deduce that

$$
\lim _{t \rightarrow \infty}\left\|\frac{1}{t} \int_{0}^{t} S(r) d r\right\|=0
$$

Next, let $f$ be a non-zero function defined on $X$, hence there exists $g \in D(A)$ such that $f=A g=-g^{\prime}$. Therefore, we obtain for $0<x \leq s$

$$
\begin{aligned}
\int_{0}^{t}(S(s) f)(x) d s & =\int_{0}^{t}(S(s) A g)(x) d s \\
& =-\int_{0}^{t}\left(S(s) g^{\prime}\right)(x) d s \\
& =-\int_{0}^{t} \int_{x}^{0} g^{\prime}(r) d r \\
& =-\int_{0}^{t}(g(0)-g(x)) d s \\
& =g(x) t .
\end{aligned}
$$

By assumption $\lim _{t \rightarrow \infty}\left\|\frac{1}{t} \int_{0}^{t} S(r) d r\right\|=0$, hence $g(x)=0$ for all $0<x \leq s$, absurd.

Finally, $\frac{1}{t} \int_{0}^{t} S(r) d r$ is divergent as $t$ tends to infinity.

Acknowledgement: The author wishes to express their indebtedness to the referee, for his suggestions and valuable comments on this paper.

\section{References}

[1] W. Arendt, Vector-valued Laplace Transforms and Cauchy Problems, Israel J. Math, 59 (3), (1987), 327-352.

[2] K. J. Engel AND R. NAGel, One-Parameter Semigroups for Linear Evolution Equations, Graduate Texts in Mathematics, vol. 194, Springer-Verlag, New York, (2000). 
[3] J. A. Goldstein, Semigroups of linear operators and applications. Oxford Mathematical Monographs. New York: Oxford University Press (1985).

[4] S. Grabiner AND J. ZemÁnek, Ascent, descent and ergodic properties of linear operators, J. Operator Theory, 48 (2002), 69-81.

[5] M. HEIBER, Laplace transforms and $\alpha$-times integrated semigroups, Forum Math. 3, (1991), 595-612.

[6] E. Hille ANd R. S. Phillips, Functional analysis and semigroups, Amer. Math. Soc. Colloq. Publ., vol. 31, Amer. Math. Soc., Providence, R. I., (1957).

[7] H. Kellerman And M. Hieber, Integrated semigroups, Journal of Functional Analysis 84, no. 1, (1989), 160-180.

[8] Y. Kozitsky, D.Shoikhet ANd J.Zemànek, Power convergence of Abel averages, Arch. Math. (Basel), 100 (2013), 539-549.

[9] U. KREngel, Ergodic Theorems, Walter de Gruyter Studies in Mathematics 6, Walter de Gruyter, Berlin-New York, (1985).

[10] M. LIN, On the uniform ergodic theorem II, Proc. Amer. Math. Soc., 46 (1974), 217-225.

[11] M. Lin, D. Shoikhet AND L. Suciu, Remarks on uniform ergodic theorems, Acta Sci. Math. (Szeged), 81 (2015), $251-283$.

[12] A. PAzY, Semigroups of Linear Operators and Applications to Partial Differential Equations, Applied Mathematical Sciences, vol. 44, Springer-Verlag, New York (1983).

[13] S.Y. SHAW, Uniform ergodic theorems for locally integrable semigroups and pseudo-resolvents, Proc. Amer. Math. Soc., 98 (1986), 61-67.

[14] S.Y. SHAW, Uniform ergodic theorems for operator semigroups, in: Proc. Anal. Conf., Singapore, 12-21 June, 1986, Amsterdam (1988), 261-265.

[15] A. Tajmouati, A. El bekkali, F. Barki and M.A. Ould Mohamed Baba, On the uniform ergodic for $\alpha$-times integrated semigroups, BSPM, (3s.) Vol. 39 No. 4, (2020) 9-20.

[16] A. Tajmouati, M. Karmouni And F. Barki, Abel ergodic theorem for $C_{0}$-semigroups, Adv. Oper. Theory Vol. 5, No 4, (2020) 1468-1479.

[17] H. R. THIEME, Integrated Semigroups and Integrated Solutions to Abstract Cauchy Problems, J. Math. Anal. Appl. 152 (1990), 416-447

[18] A.E. TAYLoR, D.C. LAY, Introduction to Functional Analysis, Wiley, New York (1980).

[19] K. YosIDA Functional analysis, 3rd ed., Springer-Verlag, New York, (1971). 